\title{
Structural Modifications of Multi-walled Carbon Nanotubes of Different Diameters through Electron Beam Irradiation
}

\author{
A. K. M. Fazle Kibria \\ International Affairs Division, Bangladesh Atomic Energy Commission, 12/A Agargaon, \\ Sher-e-Bangla Nagar, Dhaka-1207, Bangladesh
}

\begin{abstract}
The effects of irradiation on the structure of purified multi-walled carbon nanotubes (MWCNTs) having 6-19 graphitic shells and outer diameters of 8.15-17.11 nm were investigated using electron beam of energies $200 \mathrm{keV}$ and dose of $2.16 \mathrm{x} 10^{17} \mathrm{e} \mathrm{cm}^{-2} \mathrm{~s}^{-1}$. It was observed that the electron irradiation created a number of chronological alterations in the tube structures. These were identified to be tube contraction, destruction of the innermost graphitic shell, deformation of graphitic shells and its proliferation, break down of the graphitic shells and their spreading into the tube hole and finally the destruction of the whole tube. MWCNTs having the largest innermost diameter found suffer from the highest contraction. The tube contraction behavior found stops when the innermost graphitic shell starts to destroy. Irradiation affected the innermost graphitic shell first and that of the smallest diameter was the more rapidly. It occurred probably due to having the highest curvature value. Tubes having inner shell of diameter about $4.8 \mathrm{~nm}$ suffer from fractional destruction within 5-15 s of irradiation exposure. Such a shell was ruined within 1 minute of irradiation exposure but that of diameter $7.0 \mathrm{~nm}$ was survived up to 2 minutes. It seems that the irradiation induced defects created in the MWCNTs can be used for the diversified applications of nanotubes such as the hydrogen storage enhancement in them.
\end{abstract}

Keywords: Carbon nanotube, Electron irradiation, Tube contraction, Innermost shell, Defect.

\section{Introduction}

Carbon nanotubes (CNTs) have generated enormous interest among the scientists and engineers since the pioneering work done by Iijima (Iijima, 1991). These carbon structures have potential applications in the fields of nanometer power electronic devices (Baughman et al., 2002), biological sensors (Star et al., 2003), nanocomposites (Yoo et al., 2007), energy storage (Kibria et al., 2001), etc. However, to fully exploit the potentiality of CNT, effective means of tailoring CNT properties must be developed and thus various nanoengineering procedures including interaction of charged particles with CNTs have been developed (Krasheninnikov et al., 2002, Kim et al., 2004, Zacharia et al., 2005, Gomez-navarro et al., 2005, Gupta et al., 2007). Irradiation introduces a wide range of defects in CNTs and originates the existence of carbon in sp, $\mathrm{sp}^{2}$ and $\mathrm{sp}^{3}$ hybridization (Ajayn et al., 1998, Hashimoto et al., 2004, Chakraborty et al., 2007). As a result, the properties of CNTs alter which renders them to the deserved functionalities.

\footnotetext{
* Corresponding author: E-mail: kibriaf@yahoo.com
}

The mechanism of defect formation in CNTs by electron irradiation is fundamentally dependent on the displacement of C-atoms from their graphitic shells due to knock-on collision. In order to achieve these, a minimum energy known as the threshold energy $\left(T_{d}\right)$ must be transferred from an energetic electron to a surface $\mathrm{C}$-atom of nanotube (Krasheninnikov and Nordlund, 2004). Transmission electron microscope (TEM) connecting with the radiation facility was utilized for the irradiation of CNTs. The set system could create defects in CNTs by energetic electrons and concurrently monitor the induced structural changes (Krasheninnikov and Nordlund, 2004, Hashimoto et al., 2004).

Controlled irradiation seems decisive to bring CNTs in practical purposes. It was reported that electron irradiation of a MWCNT having beam energy $300 \mathrm{keV}$ and beam intensity of $450 \mathrm{~A} / \mathrm{cm}^{2}$ caused severe destruction of the tube which is not desirable for their applications (Banhart et al., 2005). On 
the other hand, electron beam $400 \mathrm{keV}$ and beam intensities of $20 \mathrm{~A} / \mathrm{cm}^{2}$ generates high defects in the graphitic shells of a MWCNT and severely disordered the tube shells within a few moments (Banhart et al., 1997). Exposure of SWCNTs in the electron beam of energies $80 \mathrm{keV}$ and $120 \mathrm{keV}$ with a dose of $1.35 \times 10^{19} \mathrm{e} \mathrm{cm}^{-2} \mathrm{~s}^{-1}$ which corresponds to a beam intensity of $2 \mathrm{~A} / \mathrm{cm}^{2}$ transformed them to amorphous form within a short exposure (Kis et al., 2004). Recently, it has been reported that a low energetic electron beam $120 \mathrm{keV}$ and a low dose of $6 \times 10^{18} \mathrm{e} \mathrm{cm}^{-2} \mathrm{~s}^{-1}$ could successfully generate the primary and secondary defects in the wall of a SWCNT without its graphitic shell destruction (Hashimoto et al., 2004). From the findings, it was predicted that diversified applications of nanocarbon materials can be carried out by taking the advantage of these instrumentally generated defects. The experimental findings has been reported by a number of authors (Banhart et al., 1997, Hashimoto et al., 2004, Banhart et al., 2005, Kis et al., 2004) as described above are clearly informing that the applied electron beam energy, set beam intensity and the irradiation time are the important factors to generate usable defects in the CNTs. Additionally, an optimization of the electron beam energy and the irradiation exposure time is a crucial factor to control the structural transformations of CNTs having different graphitic shell numbers and diameters. It should diminish the unwanted damage and destruction of CNTs during their electron irradiation dealing.

The present study reports elaborately the effects of $200 \mathrm{keV}$ energetic electron beam on the structural changes of MWCNTs having outer diameters of about 8-17 nm and graphitic shell numbers 6-19. The ability of electron irradiation to tailor the defect and destruction of graphitic shell is also described. The application feasibility of the irradiation induced defects for the enhancement of hydrogen storage in MWCNTs is briefly discussed.

\section{Materials and Methods}

Purified MWCNTs synthesized by chemical vapour deposition (CVD) technique over $\mathrm{Mo}-\mathrm{Co} / \mathrm{MgO}$ catalyst was used in this study (Kibria et al., 2004). At first, a suspension of MWCNTs in ethanol was made which was then sonicated for about $0.5 \mathrm{~h}$. Samples for the TEM studies were prepared by pouring 1-2 drops of the sonicated solution on the holey carbon coated copper grids and drying in air at room temperature. Electron beam of energy $200 \mathrm{keV}$ and dose of $2.16 \mathrm{x}$
$10^{17} \mathrm{e} \mathrm{cm}^{-2} \mathrm{~s}^{-1}$ were applied directly from the transmission electron microscope (TEM, Model 2000F, JEOL Limited, Japan, operable up to $300 \mathrm{keV}$ ) batch wise on the chosen CNTs at room temperature. The incident electron beam was allowed to cover an area of $0.14 \mu \mathrm{m}^{2}$. The employed electron beam area and the dose of electrons were $1.4 \times 10^{1}$ times and $1.3 \times 10^{4}$ times lower than that used on a MWCNT which severely destroyed it (Banhart et al., 2005). In order to obtain clear pictures on the structural transformations of irradiated MWCNTs (outer diameters $17.11 \mathrm{~nm}, 15.85 \mathrm{~nm}$ and $4.75 \mathrm{~nm}$ ), high resolution transmission electron microscopy (HRTEM) images of the irradiated tubes were recorded by a CCD-camera. Images of the irradiated tubes were recorded up to their amorphization, i.e., the destruction of the tubes.

\section{Results and Discussion}

HRTEM images of an irradiated MWCNT with respect to the period of exposure to irradiation are shown in Figures 1(a-f). For better illustration the tube is designated as 'Tube A'. The non-irradiated tube consisted of 19 graphitic shells and its outer and inner diameters were $17.11 \mathrm{~nm}$ and 4.87 nm, respectively. From Figure 1a, it may be seen that the tube has shrunk towards the interior hole within a few seconds of irradiation exposure. The extent of tube contraction within 5.5 seconds of electron irradiation was found to be 0.44-0.62 nm. During this contraction in tube, the graphitic interlayer distances remained almost equal to that of the nonirradiated tube. The measured interlayer distances are $0.34 \pm 0.02 \mathrm{~nm}$ and almost equal to the value reported earlier for that of MWCNTs (Banhart et al., 1997). From Figure 1b, it can be seen that within $15 \mathrm{~s}$ of exposure, the tube lost a fraction of its innermost graphitic shell. As a result, the inner diameter of the tube increased remarkably. No noticeable contraction of the tube occurred within this period of irradiation. On increase in irradiation exposure up to 1 minute, the tube contraction value remained almost same but that of the interior hole remarkably increased because of further loss of the remaining fraction of the innermost graphitic shell as shown in the Figure 1c. At this stage, the increase in interior hole found to be $0.71 \mathrm{~nm}$ which is equal to the sum of two graphitic layers. Within 2 minutes of irradiation exposure, numerous irradiation effects such as significant bending of the graphitic walls, decrease in inner hole diameter and the presence of enormous amounts of knocked-on carbon deposits inside the graphitic layers as marked by a circle were observed (Figure 1d). On continuation of irradiation up 

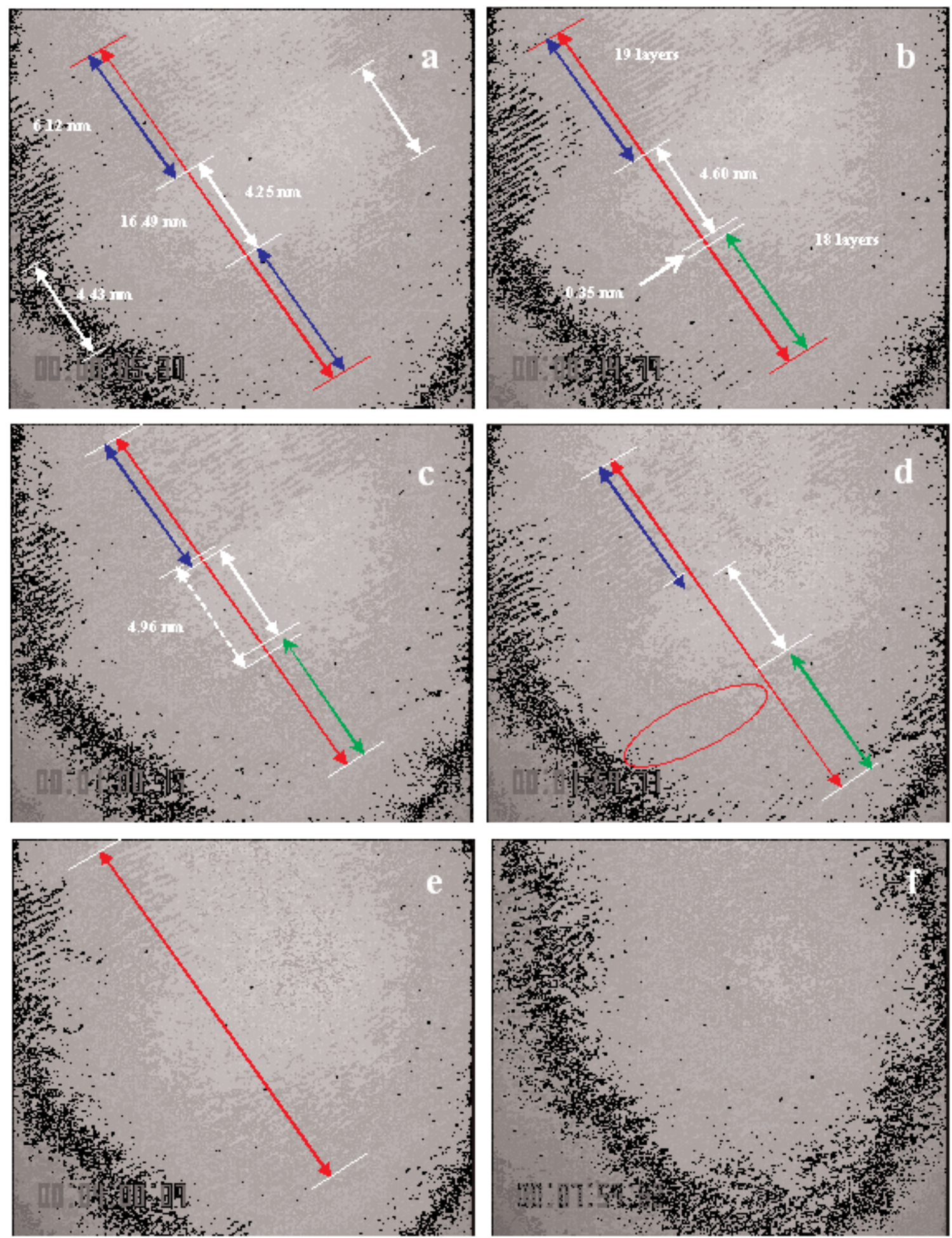

Fig. 1: High resolution transmission electron microscopy (HRTEM) images of an electron irradiated MWCNT (Tube A) with respect to the irradiation exposure time: (a) 5.5 seconds, (b) 15 seconds, (c) 1 minute, (d) 2 minutes, (e) 4 minutes and (f) 8 minutes, respectively. 

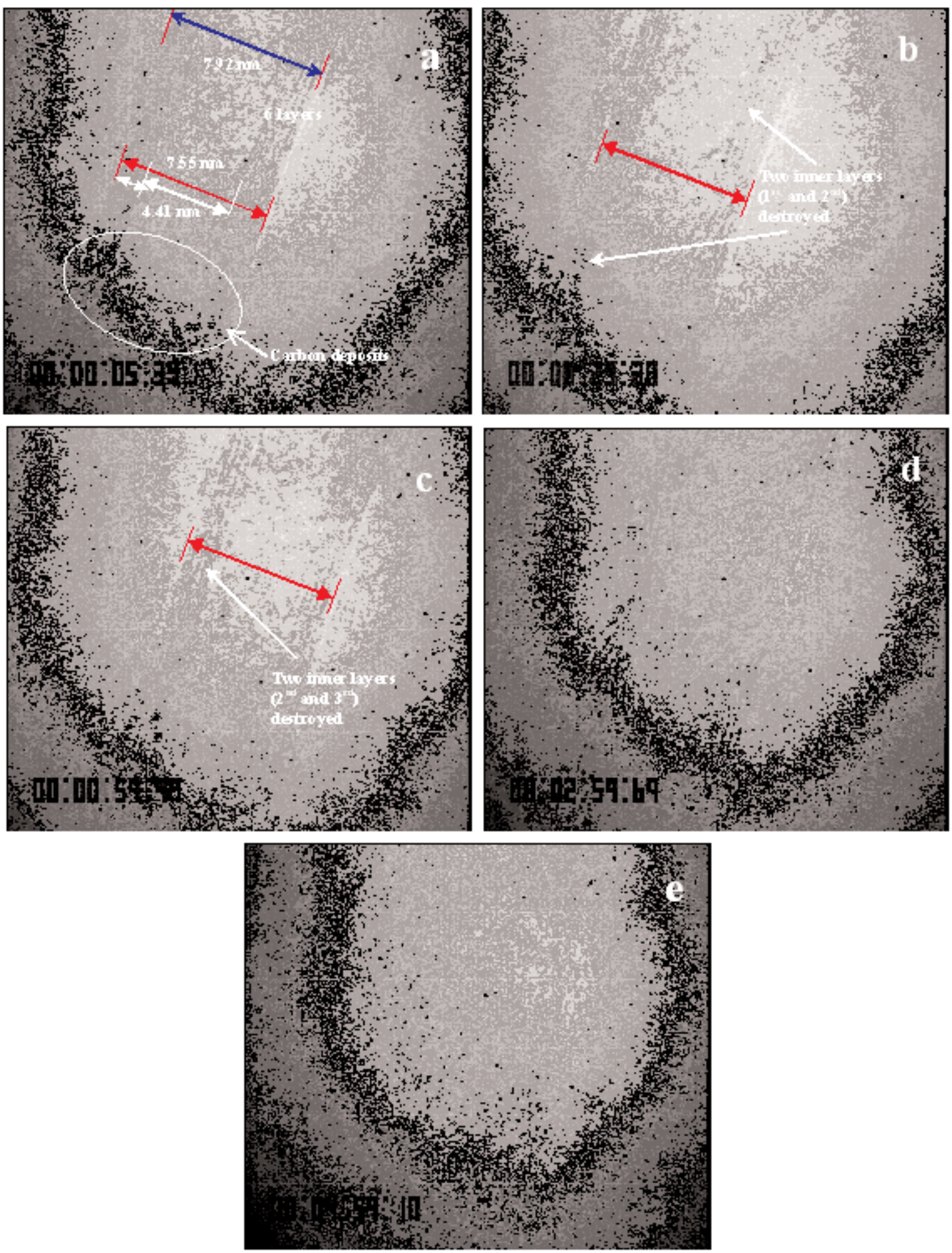

Fig. 2: High resolution transmission electron microscopy (HRTEM) images of an electron irradiated MWCNT (Tube B) with respect to the irradiation exposure time: (a) 5.6 seconds, (b) 30 seconds, (c) 1 minute, (d) 3 minutes and (e) 5 minutes, respectively 
to 4 minutes, almost all the graphitic walls has lost their ordering status as presented in the Figure 1e. On increase in irradiation time further to 8 minutes, the tube lost its graphitic structure and transformed to amorphous carbon like as shown in Figure 1f.

It was mentioned earlier that one of the objectives of the present investigation is to clearly understand the effects of electron beam irradiation on the structure of MWCNTs having different inner and outer diameters. Similar irradiation investigations as carried out on 'Tube A' were therefore carried out on two other categories of MWCNTs. Results of electron irradiation investigation of MWCNTs and the effects of irradiation exposure using electron beam of energy $200 \mathrm{keV}$ are summarized in Table I.

Figures 2(a-e) show the HRTEM images of an irradiated MWCNT designated as 'Tube B'. Its number of graphitic shells was more than three times lower and both the outer and inner diameters were comparably narrower than that of 'Tube A'. From Figure 2a, it may be seen that within $5.6 \mathrm{~s}$ of irradiation, a noticeable contraction of the tube occurred. The tube contraction value was found to be $0.23-0.60 \mathrm{~nm}$. The trend of contraction is analogous to that observed for Tube A. Fractional destruction of the innermost graphitic shell is observed clearly. Deposition of enormous amounts of knocked-on carbons can be seen in the interlayers and the interior hole of the tube. From the image $2 b$, it can be seen that the second innermost graphitic shell started to destroy within 30s of irradiation exposure. No noticeable tube contraction was occurred within this period of exposure. On increase in irradiation period up to 1 minute, the third innermost graphitic shell started to suffer from destruction as shown in Figure 2c. On continuation of irradiation up to 3 minutes, all the graphitic shells were deformed as presented in Figure 2d. From the image 2e, it is clear that the tube fully destroyed within 5 minutes of irradiation exposure.

Figures 3(a-f) show the HRTEM images of an irradiated MWCNT designated as 'Tube C'. The non-irradiated tube consisted of 14 graphitic shells. Its outer diameter stands in the midst of 'Tube A and B' but the inner diameter was more than 1.4 times higher than that of 'Tube A and B'. In this case, the extent of tube contraction within $7.5 \mathrm{~s}$ of electron irradiation found to be $0.55-1.45 \mathrm{~nm}$ as shown in Figure 3a. From the image shown in $3 \mathrm{~b}$, it can be seen that no further change in tube diameter observed on increase in irradiation exposure up to $15 \mathrm{~s}$. But on continuation of irradiation up to 1 minute, the tube diameter and that of the interior hole drastically decreased as shown in Figure 3c. The extent of tube shrinkage reached to 1.01-1.71 nm but no noticeable deformation of the graphitic shells was observed. On increase in irradiation time up to 2 minutes, the extent of tube contraction reached to about $1.7 \mathrm{~nm}$ as shown in Figure 3d. The innermost graphitic shell destroyed fractionally as marked by a rectangle. A small area of graphitic walls marked by a circle started to deform. Moreover, a little deposit of knocked-on carbon atoms from the graphitic shells holed the positions in the interlayers and also the interior hole of the tube. On continuation of irradiation up to 4 minutes, a radical change in the status of the graphitic shells was observed as shown in the Figure 3e. It may be seen that almost all the graphitic shells had lost their ordering status. Moreover, bending of graphitic walls towards the interior hole is clearly observed in a place of the tube. This bending structure decreased the tube hole remarkably. On further increase in irradiation time, the graphitic layers started to break down, began to scatter and progressively captured the total area of the interior hole. It may be seen that after 8 minutes of irradiation exposure, the tube completely destroyed and transformed to amorphous type carbon as shown in Figure 3f.

The HRTEM images presented in Figures 1-3 conclusively indicate that the set electron irradiation condition remarkably affected the structures of the MWCNTs. The sequences of the structural changes of the tubes identified to be the contraction of the graphitic shells towards the interior hole, partial and then full destruction of the innermost graphitic shell, commencement of the deformation of remaining graphitic layers, spreading of the deformed graphitic layers towards the interior hole and finally the transformation of the tube to an amorphous like structure, i.e. the destruction of the tube.

From Table I, it can be seen that the destruction period of the innermost graphitic shell of the investigated MWCNTs followed the trend of Tube $\mathrm{B}<$ Tube $\mathrm{A}<$ Tube $\mathrm{C}$. It informs that the tube having the smallest innermost diameter was affected by the electron irradiation most quickly. This happened because of exhibiting the largest curvature-induced strain in the atomic network of the innermost shell of the 'Tube A'. It was reported that due to having curvature-induced strain in the shells of CNTs, the theoretical $T_{d}$ value, i.e., the minimum initial kinetic energy of a $\mathrm{C}$-atom to escape from the graphitic wall of nanotube is quite different than that of the flat graphitic structures. The $T_{d}$ value should drop sharply 

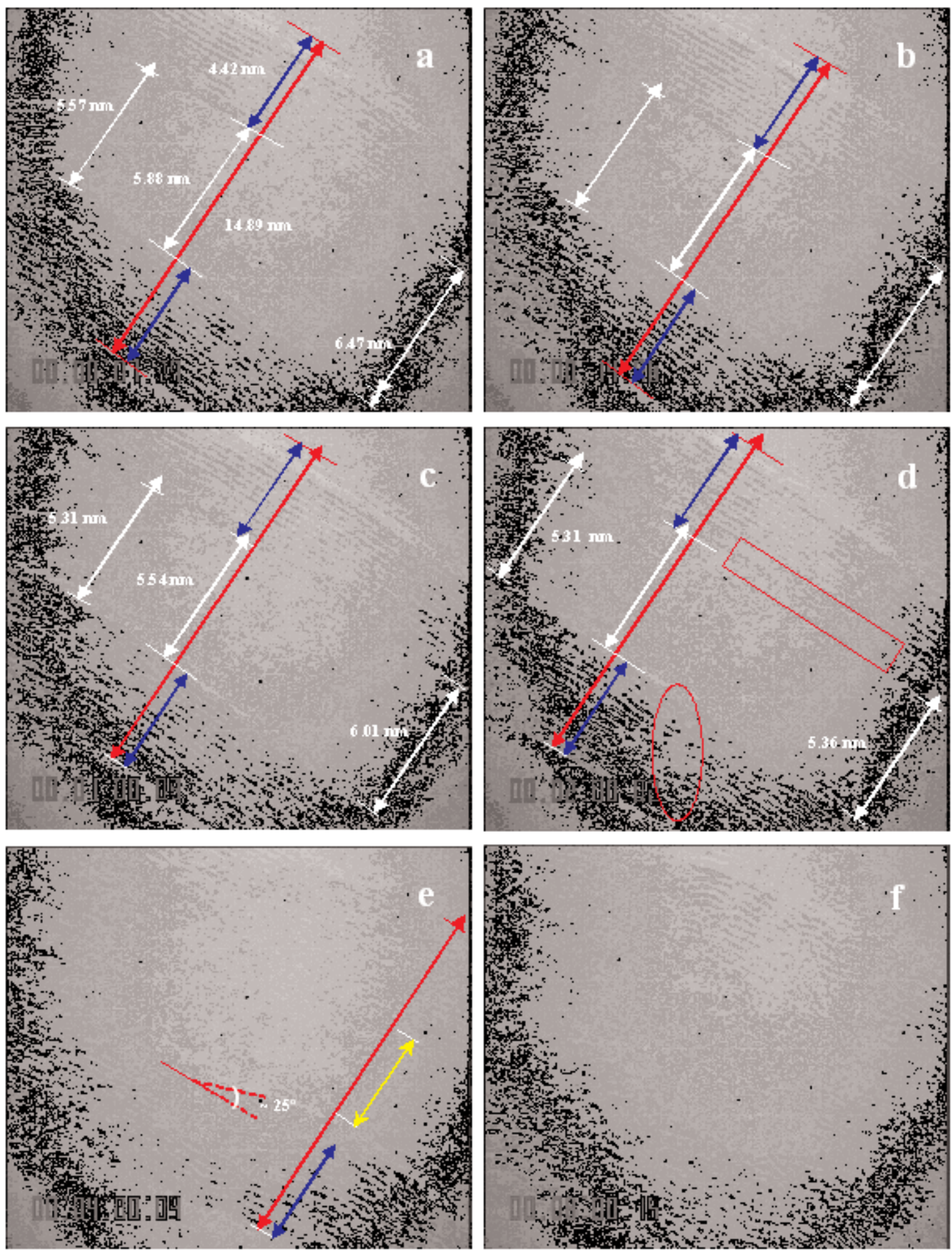

Fig. 3: High resolution transmission electron microscopy (HRTEM) images of an electron irradiated MWCNT (Tube B) with respect to the irradiation exposure time: (a) 7.5 seconds, (b) 15 seconds, (c) 1 minute, (d) 2 minutes and (e) 4 minutes and (f) 8 minutes, respectively 
Table I: Results of electron irradiation investigation of MWCNTs and the effects of irradiation exposure using electron beam of energy $200 \mathrm{keV}$

\begin{tabular}{|c|c|c|c|c|c|c|c|c|}
\hline \multirow[t]{2}{*}{$\begin{array}{l}\text { Identity of } \\
\text { tubes }\end{array}$} & \multirow[t]{2}{*}{$\begin{array}{l}\text { Outer diame- } \\
\text { ter }(\mathrm{nm})\end{array}$} & \multirow[t]{2}{*}{$\begin{array}{l}\text { Inner diame- } \\
\quad \text { ter }(\mathrm{nm})\end{array}$} & \multirow{2}{*}{$\begin{array}{l}\text { No. } \\
\text { of } \\
\text { shells }\end{array}$} & \multicolumn{3}{|c|}{$\begin{array}{l}\text { Tube contraction value }(\mathrm{nm}) \text { at dif- } \\
\text { ferent time of exposure }\end{array}$} & \multirow{2}{*}{$\begin{array}{l}\text { Innermost } \\
\text { shell destruc- } \\
\text { tion time }\end{array}$} & \multirow{2}{*}{$\begin{array}{c}\text { Number of graphitic } \\
\text { shell(s) destroyed before } \\
\text { their deformation }\end{array}$} \\
\hline & & & & 5-7.5 seconds & 1 minute & 2 minutes & & \\
\hline Tube A & 17.11 & 4.87 & 19 & $\begin{array}{c}0.44 \\
\text { to } \\
0.62 \\
\end{array}$ & - & - & $\begin{array}{c}\text { Within } \\
15 \text { seconds to } \\
1 \text { minute } \\
\end{array}$ & one \\
\hline Tube B & 8.15 & 4.75 & 6 & $\begin{array}{l}0.23 \\
\text { to } \\
0.60\end{array}$ & - & - & $\begin{array}{c}\text { Within } \\
5 \text { seconds to } \\
30 \text { seconds } \\
\end{array}$ & Two \\
\hline Tube C & 15.86 & 7.02 & 14 & $\begin{array}{l}0.55 \\
\text { to } \\
1.45\end{array}$ & $\begin{array}{l}1.01 \\
\text { to } \\
1.71\end{array}$ & $\begin{array}{l}1.66 \\
\text { to } \\
1.71\end{array}$ & $\begin{array}{l}\text { Within } 2 \text { to } \\
3 \text { minutes }\end{array}$ & Nil \\
\hline
\end{tabular}

with decreasing the tube/shell diameter because of increasing induced strain. Thus, the $\mathrm{T}_{\mathrm{d}}$ value for displacing a $\mathrm{C}$ atom is lower for a single-walled carbon nanotube (SWCNT) and correspondingly for the innermost shell of a multiwalled carbon nanotube (MWCNT) (Smith et al., 2001, Krasheninnikov and Nordlund, 2004). As for example, when the $T_{d}$ value is $15 \mathrm{eV}$, the kinetic energy of electron is equal to $82 \mathrm{keV}$ and it is very close to the experimental value 86 $\mathrm{keV}$ for SWCNTs with diameters of about $1 \mathrm{~nm}$. At electron energies $139 \mathrm{keV}$, every C-atom on a SWCNT is susceptible to ballistic ejection (Smith et al., 2001). Thereby, the innermost shell of the investigated tubes suffered knock-on

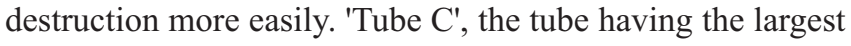
innermost diameter, showed the highest contraction value towards the interior hole. It can be seen that its contraction became insignificant when its innermost shell started to destroy. In case of other two tubes no further contraction was observed after affecting their innermost shell by irradiation. It indicates that contraction of the graphitic shells of MWCNTs stops when their innermost graphitic shell starts to suffer from destruction during the electron beam irradiation. Other mentionable finding is that MWCNTs having inner shell/shells of diameter $\sim 4.8 \mathrm{~nm}$ should suffer from fractional destruction within 5-15 s of electron irradiation at the set experimental condition. The used irradiation condition found appropriate to generate usable defects in the shells of MWCNTs having inner diameter of about $7.0 \mathrm{~nm}$. But the irradiation exposing period should be less than 2 minutes.

Presently observed contraction of the tubes is informing the generation of electron irradiation induced defects in their graphitic walls/shells. Irradiation induced defects are mainly vacancies (Ajayan et al., 1998, Hashimoto et al., 2004). Formation of vacancies usually destroys some of the stable $s p^{2}$ hybridization of the neighboring C-atoms to $s p$ type $\mathrm{C}$ atoms. Such a $s p$ type $\mathrm{C}$-atom consists of a dangling bond
(DB). Dangling bonds (DBs) are very reactive to foreign atoms like hydrogen (Lu and Pan, 2005). The DBs of a single vacancy (SV) and a double vacancy (DV) could adsorb three and four hydrogen atoms, respectively. Recently, authors (Khare et al., 2003, McDaniel et al., 2007) have reported the presence of $\mathrm{C}-\mathrm{H}$ bond in proton and $\mathrm{Ar}^{+}$beam irradiated SWCNTs. In this sense, it seems sensible to propose that the electron irradiation induced defects would contribute to improve the hydrogen storage capacity of MWCNTs for future applications.

\section{Conclusions}

An effort was taken to clearly uncover the electron irradiation effects on the structure of MWCNTs having different inner diameter and shell numbers. It was observed that the electron irradiation could induce radical changes in the structure of the tubes. Irradiation found destroyed first the innermost graphitic shells of the investigated MWCNTs. Such a shell of about $4.8 \mathrm{~nm}$ found ruins within 1 minute of irradiation but that of diameter $7.0 \mathrm{~nm}$ tolerates irradiation up to about 2 minutes. The findings could be used to process raw MWCNTs for enhancing their hydrogen storage capability.

\section{Acknowledgement}

The author is grateful to the Ministry of Education, Culture, Sports, Science and Technology (MEXT), Japan, for granting him Fellowship to carry out a part of this work in the Quantum Beam Science Directorate laboratory, Japan Atomic Energy Agency (JAEA), Tokai-mura, Japan.

\section{References}

Ajayan P. M., Ravikumar V. and Charlier J. C. (1998). Surface reconstructions and dimensional changes in single-walled carbon nanotubes. Phys. Rev. Lett., 81: 1437-40. 
Banhart F., Foiler T., Redlich P. and Ajayan P. M. (1997). The formation, annealing and self-compression of carbon onions under electron irradiation. Chem. Phys. Lett., 269: 349-55.

Banhart F. Li, J.X. and Krasheninnikov A.V. (2005). Carbon nanotubes under electron irradiation: stability of the tubes and their action as pipes for atom transport. Phys. Rev., 71B: 241408-4.

Baughman R. H., Zakhidov A. A. and Heer W. A de. (2002). Carbon Nanotubes-the route toward applications. Science, 297: 787-92.

Chakraborty A. K., Woolley R. A. J., Butenko Y. V., Dhanak, V. R., Siller L. and Hunt M. R. C. (2007). A photoelectron spectroscopy study of ion-irradiation induced defects in single-wall carbon nanotubes. Carbon, 45: 2744-50.

Gomez-navarro C., Pablo P. J de. Gomez-Herrer J., Biel B., Garcia-Vidal F. J., Rubi A. and Flores, F. (2005). Tuning the conductance of single-walled carbon nanotubes by ion irradiation in the Anderson localization regime. Nat. Mat., 4: 534-39.

Gupta S., Patel R. J., Smith N., Giedd R. E. and Hui D. (2007).Room temperature dc electrical conductivity studies of electron-beam irradiated carbon nanotubes. Diam. Relat. Mat., 16: 236-42.

Hashimoto A., Suenaga K., Gloter A.,Urita K. and Iijima S. (2004). Direct evidence for atomic defects in graphene layers. Nature (London), 430: 870-73.

Iijima S. (1991). Helical microtubes of graphitic carbon. Nature, 354: 56-8

Khare B., Meyyappan M., Moore M. H., Wilhite P. Imanaka H. and Chen B. (2003). Proton irradiation of carbon nanotubes. Nano lett., 3:643-46.

Kibria A. K. M. F., Mo Y. H., Park K. S., Nahm K. S. and Yun M. H. (2001). Electrochemical hydrogen storage behaviors of CVD, AD and LA grown carbon nanotubes in $\mathrm{KOH}$ medium. Int. J. Hydrogen Energy, 26: 823-29.

Kibria A. K. M. F, Shajahan Md., Mo Y. H., Kim M. J. and $\mathrm{Nahm} \mathrm{K.} \mathrm{S.} \mathrm{(2004).} \mathrm{Long} \mathrm{activity} \mathrm{of} \mathrm{Co}-\mathrm{Mo} / \mathrm{MgO}$ catalyst for the synthesis of carbon nanotubes in largescale and application feasibility of the grown tubes. Diam. Rela. Mat,. 13: 1865-1872.
Kim D. H., Kim C. D., Lee H. R. (2004). Effects of the ion irradiation of screen-printed carbon nanotubes for use in field emission display applications. Carbon, 42: 1807-12.

Kis A., Csányi G., Salvetat J. P., Lee T. N., Couteau E., Kulik A. J., Benoit W. Brugger J. and Fórro L. (2004). Reinforcement of single-walled carbon nanotube bundles by intertube bridging. Nat. Mat., 3:153-57.

Krasheninnikov A. V., Nordlund K., Keinonen J. and Banhart F. (2002) Ion-irradiation-induced welding of carbon nanotubes. Physical Review, 66B: 245403-6.

Krasheninnikov A. V. and Nordlund K. (2004). Irradiation effects in carbon nanotubes. Nucl. Inst. Met. Phys. Res., 216B: 355-66.

Lu A. J. and Pan B. (2005). Interaction of hydrogen with vacancies in a $(12,0)$ carbon nanotube. Phys. Rev., 71B: $165416-6$.

McDaniel F. D., Naab F. U., Holland O. W., Dhoubhadel M., Mitchell L. J. and Duggan J. L. (2007). Low-energy ion irradiation effects on hydrogen absorption and desorption in carbon nanotubes. Surf. Coat. Tech., 201: 8564-67.

Smith B. W. and Luzzi D. E. (2001). Electron irradiation effects in single wall carbon nanotubes. J. Appl. Phys., 90: $3509-15$.

Star A., Gabriel J. C. P. Bradley K. and Gruner G. (2003) Electronic detection of specific protein binding using nanotube FET devices. Nano Lett., 3: 459-63.

Yoo Y., Tuck M., Kondakindi R., Seo C. Y., Dehouche Z. and Belkacemi K. (2007). Enhanced hydrogen reaction kinetics of nanostructured Mg-based composites with nanoparticle metal catalysts dispersed on supports. $J$. Alloys Comp., 446-447: 84-9.

Zacharia R., Kim K. Y. Kibria A. K. M. F. and Nahm K. S. (2005). Enhancement of hydrogen storage capacity of carbon nanotubes via spill-over from vanadium and palladium nanoparticles. Chem. Phys. Lett., 412: 369-75.

Received : April 01, 2009;

Accepted : August 04, 2010 